\title{
Magnetic Field Effects on the Rest Potential of Ferromagnetic Electrodes
}

\author{
Gareth Hinds, Fernando M. F. Rhen, and J. M. D. Coey, Member, IEEE
}

\begin{abstract}
The application of a magnetic field unexpectedly alters the rest potential of ferromagnetic electrodes in paramagnetic solutions of their salts. Anodic shifts of up to $60 \mathrm{mV}$ in a field of 1 tesla were observed for $1 \mathrm{M}$ iron electrodes in iron nitrate solution. The magnitude of the shift depends on magnetic field strength and orientation and it varies with the anion used. The shift is smaller for cobalt and nickel $(\sim 2 \mathrm{mV})$ than for iron. The effect is attributed to modifications in the rate of mass transport under the influence of the magnetic field gradient force.
\end{abstract}

Index Terms-Electrode, ferromagnetic, field gradient force, magnetic field, magnetoelectrochemistry, rest potential.

\section{INTRODUCTION}

M AGNETIC field effects on electrochemical reactions may be divided into three categories; those on mass transport, electrode kinetics, and electrochemical equilibria. Of these, field effects on mass transport have been most widely studied [1]. An applied magnetic field can influence the transport of electroactive species in solution in a number of different ways depending on experimental conditions [2]. Magnetic forces give rise to convection in the solution which narrows the diffusion layer, resulting in higher currents. The most significant magnetic forces are the Lorentz force, $\mathbf{F}_{\mathbf{L}}$, which arises from the interaction between the magnetic field and the current [3], [4], and the field gradient force, $\mathbf{F}_{\mathbf{B}}$, which tends to move paramagnetic ions along the field gradient [5].

The effect of a magnetic field on the kinetics of electrode reactions is more controversial, with the majority of authors reporting no change in various kinetic parameters in the presence of the field [6].

Effects of magnetic fields on electrochemical equilibria are unexpected given that the magnetic energy, $\mu_{B} B$, is nearly three orders of magnitude smaller than the thermal energy at room temperature. Even for a ferromagnetic material, the magnetic energy per mole, $B M V_{m}$, where $M$ is the magnetization $(\sim$ $\left.10^{6} \mathrm{~A} / \mathrm{m}\right)$ and $V_{m}$ is the molar volume $\left(10^{-5} \mathrm{~m}^{3}\right)$ is $0.1 \mathrm{~kJ} / \mathrm{mol}$ in high fields $(B \sim 10 T)$, a factor of 25 smaller than the thermal energy. However, in recent years, several authors have reported shifts in the rest potential of ferromagnetic electrodes such as iron in paramagnetic solutions of their salts [7]-[9]. Waskaas and Kharkats reported shifts of up to $80 \mathrm{mV}$ in the rest

Manuscript received February 13, 2002; revised May 13, 2002. This work was supported in part by Enterprise Ireland under Contract ST/1999/181 "Magnetic Electroplating" and in part by Science Foundation Ireland.

The authors are with the Physics Department, Trinity College, Dublin 2, Ireland (e-mail: ghinds@tcd.ie; rhenf@tcd.ie; jcoey@tcd.ie).

Digital Object Identifier 10.1109/TMAG.2002.802506. potential of an iron electrode immersed in various paramagnetic iron salt solutions [7]. Even more surprisingly, they found that whereas the shift was in the anodic direction for iron, the potential shifted cathodically for both cobalt and nickel. No effect was observed for diamagnetic solutions or for nonferromagnetic electrodes in any solution. The authors explained these results in terms of convective transport of the cations under the influence of the paramagnetic gradient force, $\mathbf{F}_{\mathbf{P}}$. This force arises from the gradient in magnetic susceptibility due to the concentration gradient in the diffusion layer, which is created by the corrosion current. Perov et al. observed similar results for iron, but reported no effect whatsoever for cobalt and nickel [9]. Their conclusion was that the domain wall density at the electrode surface somehow drives the field effect.

Faced with these conflicting reports, the aim of this paper was to confirm whether the effects did indeed exist and to identify a likely mechanism.

\section{EXPERIMENTAL METHODS}

A two-electrode electrochemical cell was used. The working electrode was iron, cobalt, or nickel as appropriate and the reference electrode was $\mathrm{Ag} / \mathrm{AgCl}(3 \mathrm{M} \mathrm{NaCl})$. Solutions were prepared using salts of analytical grade in triply distilled water. The electrolyte concentration was $1 \mathrm{M}$ in all cases. Electrodes were made from iron, cobalt, or nickel foil or wire as appropriate. The foil electrodes were of dimensions $5 \mathrm{~mm} \times 5 \mathrm{~mm}$ $\times 0.5 \mathrm{~mm}$ and were attached to copper wire using silver paint. The back and sides of these electrodes were coated with varnish. The diameter of the wire electrodes varied from $1 \mathrm{~mm}$ down to $50 \mu \mathrm{m}$. They were coated in varnish and cut to expose a clean face. All electrodes were prepared by polishing with fine grain sandpaper and rinsing in triply distilled water. For each experiment, the working electrode was fixed to the side of the reference electrode using Kapton tape. An electromagnet was used to apply a highly uniform field of up to 1.4 tesla (T) over the entire cell. The field inhomogeneity was less than $0.01 \%$ over the cell volume. The field could be applied either parallel or perpendicular to the face of the working electrode. In a typical experiment the field was swept up to $1.4 \mathrm{~T}$ and back to zero at a constant rate of $0.1 \mathrm{~T} / \mathrm{s}$ and the open circuit potential was monitored as a function of time using a multimeter. In the case of cobalt and nickel, it was necessary to use an ac technique to measure the potential shift. Here, an alternating field with a frequency of $0.2 \mathrm{~Hz}$ was applied to the cell and the response was monitored using a Perkin Elmer 7265 lock-in detector. 


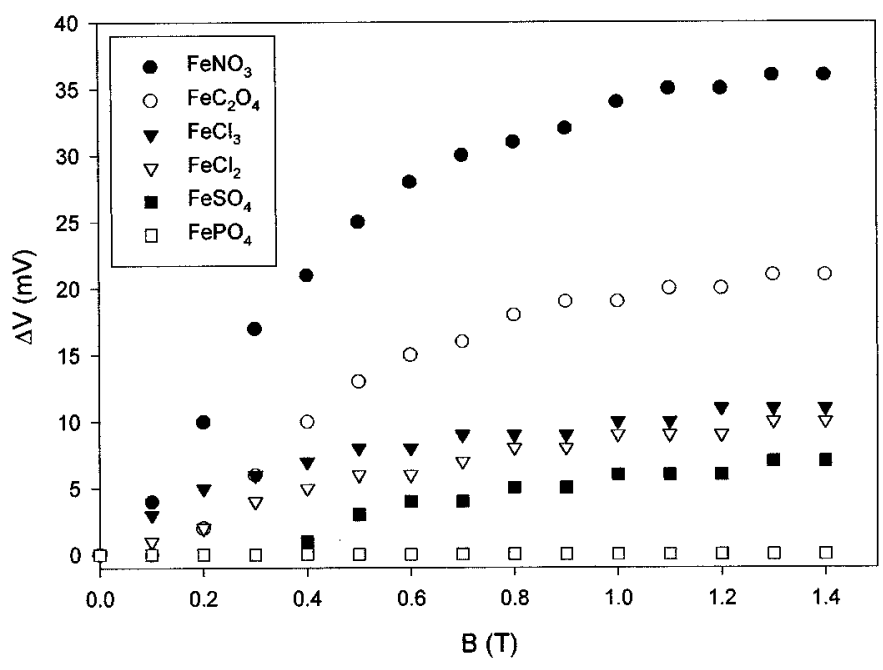

Fig. 1. Potential shift as a function of applied magnetic field for an $1 \mathrm{M}$ iron electrode in various iron salt solutions. The field is applied parallel to the electrode surface.

\section{RESULTS}

The rest potential of an iron electrode in various iron salt solutions is shown as a function of applied magnetic field in Fig. 1. The working electrode is a piece of iron foil of surface area $5 \mathrm{~mm}$ $\times 5 \mathrm{~mm}$. The electrolyte concentration is $1 \mathrm{M}$ and the field is applied parallel to the electrode surface. The shift in potential in the field is strongly dependent on the anion used and is greatest for iron (III) nitrate ( $35 \mathrm{mV}$ in $1.4 \mathrm{~T})$. In all cases the shift is anodic in direction. It was noted that in the absence of any applied field, an equivalent anodic shift could be obtained by agitating the solution. No effect was observed for an iron electrode in a diamagnetic solution $(\mathrm{AgCl})$, or for nonmagnetic electrodes $(\mathrm{Cu}, \mathrm{Zn})$ in any solution. No effect was observed for stainless-steel electrodes (both magnetic and nonmagnetic grades).

The potential shift for cobalt and nickel was an order of magnitude lower. Anodic shifts of approximately $2 \mathrm{mV}$ in $1 \mathrm{~T}$ were observed for each metal, but the presence of significant drift in the rest potential in zero field meant that it was necessary to use the ac technique. When a sinusoidal magnetic field of amplitude $0.45 \mathrm{~T}$ and frequency $0.2 \mathrm{~Hz}$ was applied to the cell, the response measured by the lock-in detector was $0.8 \mathrm{mV}$ for cobalt and $0.7 \mathrm{mV}$ for nickel (both in nitrate solution).

The remaining experiments were carried out using iron electrodes, made from high purity iron foil or wire, in iron (III) nitrate solution, as this system showed the largest effects. These electrodes showed a maximum shift of around $60 \mathrm{mV}$ in a parallel field of $1 \mathrm{~T}$ for both foil and wire electrodes. The shift remained at $60 \mathrm{mV}$ for wire diameters in the range $1 \mathrm{~mm}$ to $50 \mu \mathrm{m}$. However, no potential shift was observed for very smooth iron electrodes produced by electrodeposition.

The effect of field orientation was investigated using $1 \mathrm{~mm}$ diameter iron wire. The potential shift decreased from $60 \mathrm{mV}$ when the field was applied parallel to the surface of the electrode to $20 \mathrm{mV}$ in a perpendicular field.

The potential shift was monitored in real-time using a Solartron 1287 potentiostat to record the open circuit potential. A typical transient for an iron wire electrode in $1 \mathrm{M}$ iron (III)

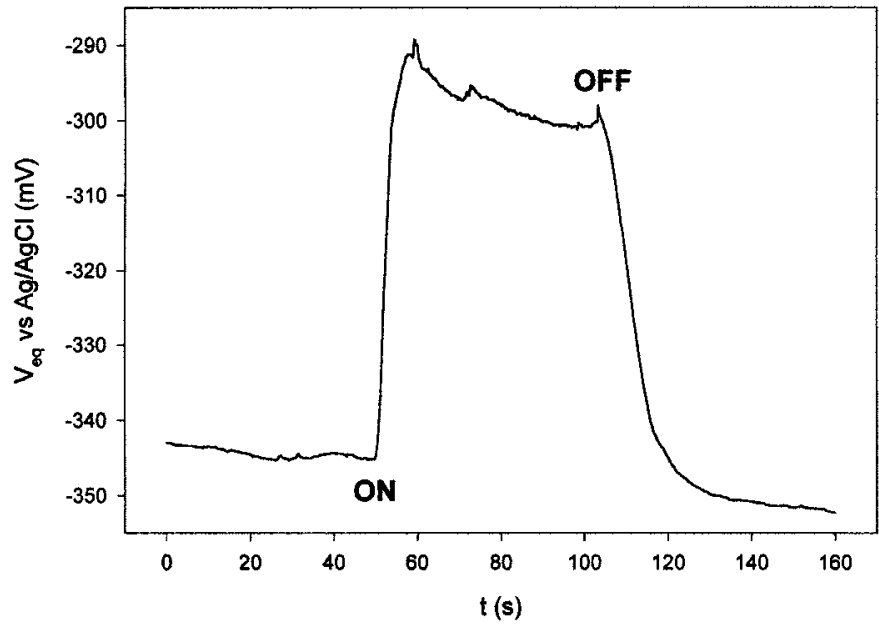

Fig. 2. Variation of rest potential as a function of time while the magnetic field is switched $\mathrm{ON}$ and OFF again. The working electrode is iron and the electrolyte $1 \mathrm{M}$ iron (III) nitrate solution. The field (1 T) is applied parallel to the electrode surface.

nitrate is shown in Fig. 2. A sharp change in potential is observed when the field is stepped to $1 \mathrm{~T}$ and a similar sharp drop is observed when the field is switched off. In between, while the field is still on, a slow decrease in potential is observed. If the field is left on, the potential returns to its original value after about $15 \mathrm{~min}$.

Once the electrode has been in the solution for a while, a dark layer forms on the surface due to corrosion. When this happens, the magnitude of the potential shift is reduced (typically by up to $50 \%$ ). When this layer is removed by abrasion, the potential shift reverts to its original value.

It was confirmed that any induced electrommotive due to the time-varying magnetic field during the experiment were negligible with respect to the measured potentials. The average emf induced in the cell was $10 \mu \mathrm{V}$ with a sweep rate of $0.1 \mathrm{~T} / \mathrm{s}$.

\section{DISCUSSION}

The results shown above demonstrate that there is a significant effect of magnetic field on the rest potential of ferromagnetic electrodes in paramagnetic solutions. The shift is anodic for all three metals $(\mathrm{Fe}, \mathrm{Co}, \mathrm{Ni})$, in contrast to the report of Waskaas and Kharkats [7].

From a thermodynamic perspective, these results are entirely unexpected. The energy shifts due to the ferromagnetic magnetization in $1 \mathrm{~T}$ are $0.14 \mathrm{mV}(\mathrm{Fe}), 0.10 \mathrm{mV}(\mathrm{Co})$, and $0.04 \mathrm{mV}$ (Ni).

It is clear then that the effect must have its origin in magnetic field induced convection of the electrolyte solution. This is supported by the observation that an equivalent effect is produced by agitating the solution. There are several magnetic body forces which could be responsible for this induced convection [2]. Two of these are related to the magnetic properties of the electrolyte. The energy, $E_{\mathrm{mag}}$, of the electrolyte in the magnetic field is $-(1 / 2) M B\left(\mathrm{~J} / \mathrm{m}^{3}\right)$ where $M(\mathrm{~A} / \mathrm{m})$ is the magnetization induced by the field $B(\mathrm{~T})$. Now, $M=\chi_{m} c B / \mu_{0}$, where $\chi_{m}$ is the molar susceptibility, hence

$$
E_{\mathrm{mag}}=-\chi_{m} c B^{2} / 2 \mu_{0} \text {. }
$$


The related force $\mathbf{F}_{\text {mag }}=-\nabla E_{\text {mag }}$ includes two terms

$$
\mathbf{F}_{\text {mag }}=\chi_{m} B^{2} \nabla \mathbf{c} / 2 \mu_{0}+\chi_{m} c B \nabla \mathbf{B} / \mu_{0} .
$$

The first term is the paramagnetic gradient force, $\mathbf{F}_{\mathbf{P}}$

$$
\mathbf{F}_{\mathbf{P}}=\chi_{m} B^{2} \nabla \mathbf{c} / 2 \mu_{0}
$$

which arises from the variation in the paramagnetic susceptibility of the diffusion layer due to the concentration gradient of the cations there. The second term is the field gradient force, $\mathbf{F}_{\mathbf{B}}$

$$
\mathbf{F}_{\mathbf{B}}=\chi_{m} c B \nabla \mathbf{B} / \mu_{0}
$$

which is the force due to the field gradient, $\nabla \mathbf{B}$, in the solution when the field is nonuniform.

The Lorentz force, $\mathbf{F}_{\mathbf{L}}$, and the paramagnetic gradient force, $\mathbf{F}_{\mathbf{P}}$, cannot be responsible for the induced convection here, since, if this were the case, the effect would not be limited to ferromagnetic electrodes. Furthermore, the paramagnetic gradient force is unlikely to influence mass transport because it acts along the same line as the much larger driving force for diffusion [2].

The outstanding features of the data are that the effect is limited to 1) ferromagnetic electrodes and 2) paramagnetic solutions. Both of these features together suggest that the most likely source of convection is, therefore, the field gradient force, $\mathbf{F}_{\mathbf{B}}$. The field gradient arises from the stray field close to the magnetized electrode surface and exerts a force on the paramagnetic species in solution. The anodic potential shift may then be caused by the enhancement in mass transport for the cathodic corrosion current. This is supported by the fact that the effect is only observed for corroding systems and would explain why the potential shift for iron is an order of magnitude higher than for cobalt and nickel. It would also account for the largest shifts occurring in nitrate solution.

An alternative explanation is that the surface concentration, $c_{0}$, of cations is increased by the action of the field gradient force. The equilibrium potential, $V_{e q}$, is determined by the Nernst equation

$$
V_{e q}=V^{0^{\prime}}+R T \ln c_{0} / n F
$$

where $V^{0^{\prime}}$ is the formal potential. The Nernst equation shows qualitatively that the rest potential will shift anodically if the sur- face cation concentration is increased. Grant et al. have showed that it is possible to trap paramagnetic ions at magnetized ferromagnetic microelectrodes under the action of the field gradient force [10]. It is possible that a similar mechanism is operating here. The slow restoration of the original potential while the field is still on, shown in part in Fig. 2, may be related to the gradual escape of such trapped cations under thermal influences.

Experiments using lithographically patterned electrodes to achieve a controlled variation in surface roughness are required to determine conclusively the role played by the stray field in inducing convection in these systems.

\section{CONCLUSION}

The rest potential of ferromagnetic electrodes in paramagnetic solutions of their salts is modified by an applied magnetic field. The shift is anodic in direction for iron, cobalt, and nickel. The effect is attributed to modifications in the rate of mass transport due to the field gradient force, $\mathbf{F}_{\mathbf{B}}$.

\section{REFERENCES}

[1] R. A. Tacken and L. J. J. Janssen, "Applications of magnetoelectrolysis," J. Appl. Electrochem., vol. 25, pp. 1-5, 1995.

[2] G. Hinds, J. M. D. Coey, and M. E. G. Lyons, "Influence of magnetic forces on electrochemical mass transport," Electrochem. Commun., vol. 3, pp. 215-218, 2001.

[3] R. Aogaki, K. Fueki, and T. Mukaibo, "Application of magnetohydrodynamic effect to the analysis of electrochemical reactions-1. MHD flow of an electrolyte solution in an electrode-cell with a short rectangular channel," Denki Kagaku, vol. 43, pp. 504-508, 1975.

[4] - "Application of magnetohydrodynamic effect to the analysis of electrochemical reactions-2. Diffusion process in MHD forced flow of electrolyte solutions," Denki Kagaku, vol. 43, pp. 509-514, 1975.

[5] S. R. Ragsdale, K. M. Grant, and H. S. White, "Electrochemically generated magnetic forces, enhanced transport of a paramagnetic redox species in large, nonuniform magnetic fields," J. Amer. Chem. Soc., vol. 120, pp. 13461-13468, 1998.

[6] O. Devos, O. Aaboubi, J.-P. Chopart, A. Olivier, C. Gabrielli, and B. Tribollet, "Is there a magnetic field effect on electrochemical kinetics?," J. Phys. Chem. A, vol. 104, pp. 1544-1548, 2000.

[7] M. Waskaas and Y. I. Kharkats, "Magnetoconvection phenomena: A mechanism for influence of magnetic fields on electrochemical processes," J. Phys. Chem. B, vol. 103, pp. 4876-4883, 1999.

[8] N. S. Perov, A. V. Bozhkov, and A. A. Radkovskaya, "An electro-chemical magnetic field sensor," Sensors Actuators A, vol. 81, pp. 351-354, 2000.

[9] N. S. Perov, P. M. Sheverdyaeva, and M. Inoue, "Effect of magnetic field on the electrode potential of metals," J. Appl. Phys., vol. 91, pp. 8557-8559, 2002.

[10] K. M. Grant, J. W. Hemmert, and H. S. White, "Magnetic focusing of redox molecules at ferromagnetic microelectrodes," Electrochem. Commun., vol. 1, pp. 319-323, 1999. 